\title{
Carboxymethyl starch as a polymeric plant based excipient in drug delivery
}

\author{
Thayyath Sreenivasan Anirudhan ${ }^{凶}$ and Jayasree Parvathy
}

\begin{abstract}
Polysaccharides extracted and isolated from plant products serve as potential candidates in pharmaceutics mainly drug delivery, owing to its biodegradability, bioavailability and non-toxic character. Hydrogels, three-dimensional hydrophilic polymeric networks, exhibit dramatic changes in their swelling behaviour, network structure, and mechanical strength in response to different stimuli to the body. A wide variety of polysaccharides (chitosan, alginate, cellulose, starch, etc) and their derivatives have been used to synthesize hydrogels that tend to swell in water or biological fluids. Such systems have numerous biomedical applications including: drug delivery, wound dressing, and tissue engineering. However, single-network hydrogels have weak mechanical properties and slow response at swelling. To enhance the mechanical strength and swelling/deswelling response of hydrogels, multicomponent networks as interpenetrating polymer networks have also been reported and recently have gained much importance in research on different biomedical applications. The present review focuses on the application of carboxymethyl starch as a pharmaceutical excipient where the effect of crosslinking modification and the formation of Semi Interpenetrating Polymer Network with montmorillonite clay mineral have been investigated.
\end{abstract}

Keywords: carboxymethyl starch; hydrogels; semi interpenetrating polymer network; excipient; drug delivery

Received: 25 April 2014 / Accepted revised version: 07 July 2014 / Published online: 01 October 2014

(C) Horizon e-Publishing Group

CITATION

Anirudhan, T. S., \& Parvathy, J. (2014). Carboxymethyl starch as a polymeric plant based excipient in drug delivery. Plant Science Today, 1(4), 179-182. http://dx.doi.org/10.14719/pst.2014.1.4.38

AUTHOR'S AFFILIATION

Department of Chemistry, University of Kerala, School of Physical and Mathematical Sciences, Kariavattom, Trivandrum-695 581, India

CORRESPONDENCE

$\square$ Prof. T.S. Anirudhan, Email: tsani@rediffmail.com

\section{Introduction}

Mother Nature has gifted us wide variety of materials around us to improve and sustain the health of all living beings directly or indirectly. Starch is a natural, cheap, available, renewable, and biodegradable polymer produced by many plants as a source of stored energy. It is a natural polymer regenerated from carbon dioxide and water by photosynthesis in plants (Teramoto, Motoyama, Yosomia, \& Shibata, 2003). It is found in plant leaves, stems, roots, bulbs, nuts, stalks, crop seeds, and staple crops such as rice, corn, wheat, cassava, and potato. It is either used as extracted from the plant and is called "native starch", or it undergoes one or more modifications to reach specific properties and is called "modified starch". Owing to its attractive combination of availability and price, it is suitable for the production of biopolymer and has been considered as one of the most promising candidate for biomedical applications. The main sources of starch include maize $(80 \%)$, wheat $(8 \%)$, potatoes $(5 \%)$, cassava (5\%), jackfruit (2\%). Starch was added to rye and wheat breads during the 1890s in Germany and to beer in 1918 in England. Also, Moffett, writing in 1928, had described the use of corn starch in baking powders, pie fillings, sauces, jellies and puddings. The 1930s saw the use of starch as components of salad dressings in mayonnaise. Subsequently, combinations of corn and tapioca starches were used by salad dressing manufacturers (Mason, 2003).

\section{Carboxymethyl starch}

Native starch may exhibit some drawbacks that restrict its use such as sensitivity to shearing and low $\mathrm{pH}$, thermal resistance, high tendency towards retrogradation, brittleness and lack of specific functional groups that may interact with different groups or substances (Angellier, Choisnard, Molina-Boisseau, Dole, \& Dufresne, 2004). Therefore the chemical modification offers an interesting alternative to develop new materials, providing biodegradation properties. Chemical modification of starch involves reaction of the hydroxyl groups on the anhydroglucose units (AGU) and these have been used to produce starch derivatives based on carboxymethylation 
(Bi, Liu, Wu, \& Cui, 2008), oxidation (Tolvanen, Mäki-Arvela, Sorokin, Salmi, Murzin, 2009), grafting (Mishra, Mukul, Sen, \& Jha, 2011), hydroxypropylation (Kim, Choi, Kim, \& Baik, 2011) and crosslinking (Gunaratne, \& Corke, 2007; Nagasawa, Yagi, Kume, \& Yoshii, 2004). It has been shown that, chemically modified starches have more reactive sites to carry biologically active compounds, they become more effective biocompatible carriers and can easily be metabolized in the human body (Prochaska, Konowal, Sulej-Chojnacka, Lewandowicz, 2009). Among these starch derivatives, carboxymethyl starch (CMS) as a kind of etherified starch has attracted a lot of attention in both research and industry (Lawal, Lechner, Hartmann, \& Kulicke, 2007). The presence of functional group $\left(-\mathrm{CH}_{2} \mathrm{COO}^{-}\right)$yields starch with many unique properties, such as low gelatinization temperature, excellent flexibility, improved paste storage stability and clarity (Qiu \& He, 1999).

Carboxymethyl starch is synthesized by reacting starch with monochloroacetic acid or its sodium salt after activation of the polymer with aqueous $\mathrm{NaOH}$ in slurry of an aqueous organic solvent, mostly an alcohol. CMS is an example of esterified starch. The hydroxyl groups of starch molecules were etherified by carboxymethyl groups. In carboxymethylation, starch was reacted with sodium monochloroactate in the presence of $\mathrm{NaOH}$. The formation of Carboxymethyl starch is depicted in Fig. 1.
The efficiency of the reaction was determined by degree of substitution. The carboxymethylation of starch progressively increases water solubility. As the degree of substitution increases, the modified starch is more soluble in the cold water yielding a clear solution. Carboxymethyl starch acts as an anionic polyelectrolytic in an aqueous slurry or paste. The rate of swelling to reach peak viscosity and cooked out viscosity of modified starch is higher than the native starch. CMS paste is more cohesive and tendency to gel. The retrogradation is greatly diminished.

\section{Drug Delivery Systems}

Drug delivery System (DDS) is defined as the method or process of administering a pharmaceutical compound to achieve therapeutic in human or animals. DDS are required for the drugs with narrow therapeutic concentration range of blood circulation and eliminate rapidly or maintaining the concentration below levels where potential harmful side effects become prevalent. DDS have the advantage of increasing the residence time of drug with in a patient, reducing the dosage frequency and toxic effects, improving patient compliance.

\section{Carboxymethyl starch based matrices as effective drug carriers}

The application of polymeric materials for medical purposes is growing vastly as modern advances in drug delivery are now predicted upon the rational design of

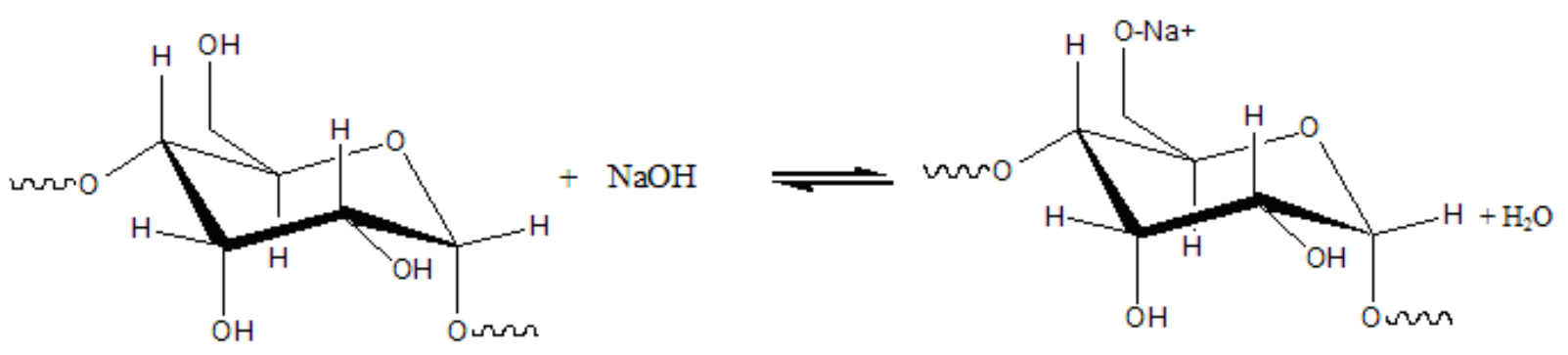

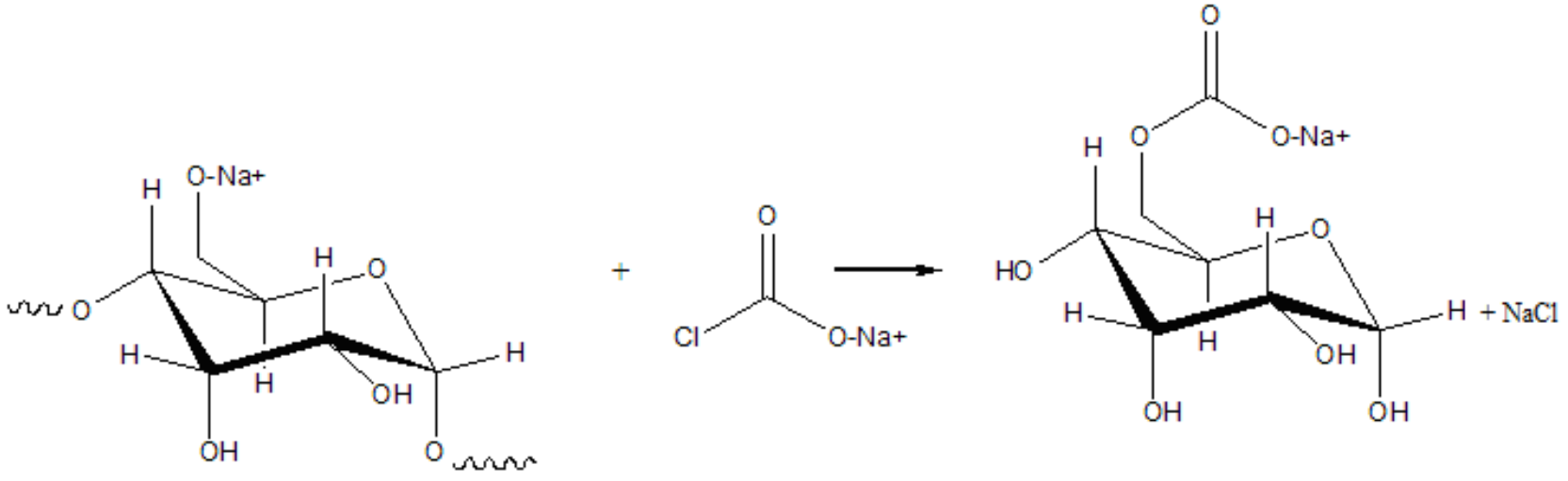

Fig 1. Overview of the synthetic procedure for the formation of Carboxymethyl starch 
polymers tailored to exert distinct biological functions. A number of degradable polymers are potentially useful for this purpose, including a variety of synthetic and natural substances.

Carboxymethyl mungbean starch (SCMMSs) exhibited the ability to form a clear, thin film with greater flexibility and strength than that of native starch which makes it potential for use as table film coating agent (Kittipongpatana et al., 2006). In vitro and in vivo evaluation of High-amylose carboxymethyl starch matrices for oral and sustained release of Acetaminophen was conducted (Nabais, Brouillet, Kyriacos, \& Mroueh, 2007). Polymeric material based on Carboxymethyl starch and the controlled release of Aspirin was conducted where the matrix released the enclosed drug at a much faster rate in neutral and alkaline $\mathrm{pH}$ than in acidic $\mathrm{pH}$, thus holding the promise of the targeted delivery of the drug to the gastrointestinal tract (Sen \& Pal, 2009). ChitosanCarboxymethyl starch nanoparticles were developed by complex coacervation process for the controlled release of 5-aminosalicylic acid.

Adding bulky functional groups like carboxymethyl group reduces the tendency of starch to recrystallize and makes it less prone to damage by heat and bacteria. Carboxymethylation results in the modification of starch due to the introduction of carboxylate groups that disturb the ordered structure of native starch, increase solubility in water and lead to higher storage stability. An Interpenetrating Polymer Network (IPN) is a polymer comprising two or more networks which are at least partially interlaced on a molecular scale but not covalently bonded to each other and cannot be separated unless chemical bonds are broken where as a Semi-IPN is a polymer comprising one or more networks and one or more linear or branched polymer(s) characterized by the penetration on a molecular scale of at least one of the networks by at least some of the linear or branched macromolecules. The previous investigations are mainly focused on the Semi-IPN systems of organic components (Wang, Zhou, \& Xiao, 2013). However, the studies on organic-inorganic hybrid Semi- IPNs remain largely unexplored. Organic-inorganic hybrid Semi-IPNs combine the advantages of organic materials with those of inorganic polymers. Montmorillonite is a very soft phyllosilicate mineral that typically forms in microscopic crystals, forming clay. Montmoillonite, commonly known as medical clay swells with the addition of water. However, some montmorillonite expands considerably more than other clays due to water penetrating the interlayer molecular spaces and concomitant adsorption. The amount of expansion is due largely to the type of exchangeable cation
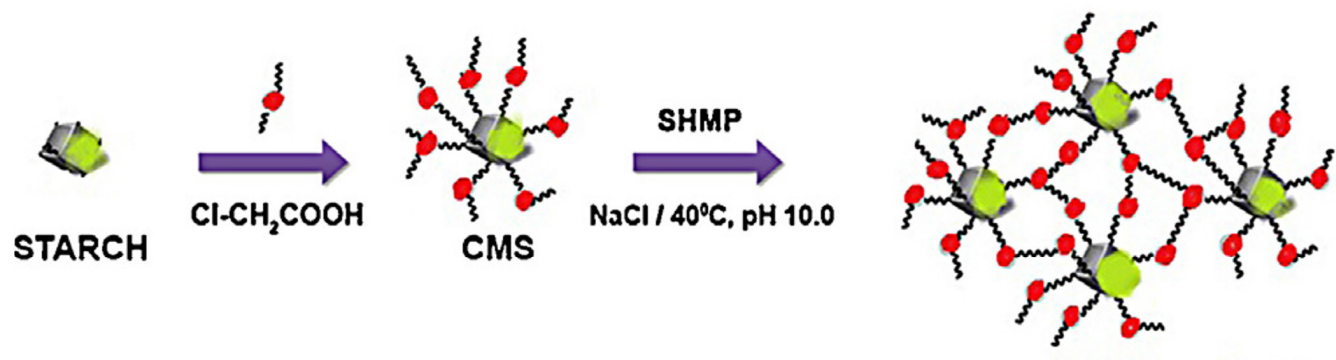

CL-CMS

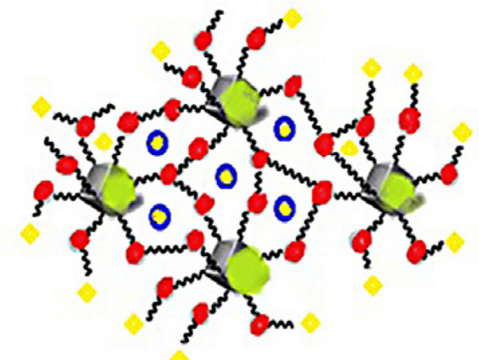

(SEMI-IPN)-DRUG

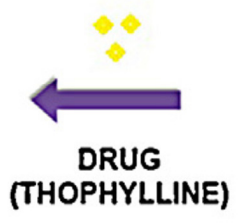

Fig. 2. Schematic representation of CL-CMS/MMT Semi-IPN system 
can result in the clay swelling to several times its original volume.

As carboxymethyl starches also possess the tendency of aggregation similar to native starches, our research group mainly focused attempts to crosslink CMS with sodium hexa meta phosphate (SHMP) and a novel Semi-IPN drug delivery system comprising crosslinked carboxymethyl starch and Montmorillonite (CL-CMS)/MMT for the controlled release of Theophylline. Fig. 2 shows the schematic representation of the Semi-IPN drug delivery system (Anirudhan \& Parvathy, 2014).

It is designated to assess the effectiveness of the drug delivery vehicle in the encapsulation of Theophylline. Theophylline, known as dimethylxanthine, is a methylxanthine drug used in therapy for respiratory diseases such as COPD and asthma under a variety of brand names. As a member of the xanthine family, it bears structural and pharmacological similarity to caffeine. The main actions of theophylline involve relaxation of bronchial smooth muscles and increasing heart muscle contractility and efficiency. The use of theophylline is a bit complicated because of its interaction with various drugs and that it has a narrow therapeutic index. So the controlled and sustained delivery of theophylline is administered as effective medication to treat asthma and other respiratory diseases.

\section{Conclusions}

Controlled drug delivery devices that utilize biodegradable polymers are enjoying high resurgence of interest, as there is no need for the surgical removal of the device. The use of natural polymers as drug carriers has received much attention in the pharmaceutical field due to their biocompatibility and safety. Biodegradable natural polymers as drug carriers possess many advantages including good biocompatibility, non toxicity and controlled release properties. The actual tendency is directed to the development of materials that are assimilated by the organism producing minimum collateral effects. Controlled release technology has shown important potentials in the field of medicine, pharmacy and agriculture. Controlled or sustained release drugs provide many advantages in comparison with conventional forms such as reducing side effects, keeping drug concentration at effective levels in plasma, improving utilization of the drug and decreasing the dosing. Our present study is designated to assess the effectiveness of the drug delivery vehicle in the encapsulation of drugs. Several Polymers from plant origin have been successfully used and investigated as matrices in the design of polymeric biomaterial for effective drug delivery. Plants provide an attractive and non-renewable source not only for active pharmaceutical ingredients but also for architecturing materials for controlled and targeted drug delivery.

\section{References}

Angellier, H., Choisnard, L., Molina-Boisseau, S., Dole, P., \& Dufresne, A. (2004). Biomacromolecules, 5, 1545-1551. http://dx.doi.org/10.1021/bm049914u PMid:15244476

Anirudhan, T. S., \& Parvathy, J. (2014). International Journal of Biological Macromolecules, 67, 238-245. http://dx.doi.org/10.1016/i.ijbiomac.2014.03.041 PMid:24685463

Bi, Y., Liu, M., Wu, L., \& Cui, D. (2008). Polymers for Advanced Technologies, 19, 1185-1192. http://dx.doi.org/10.1002/pat.1102

Gunaratne, A., \& Corke, H. (2007). Carbohydrate Polymers, 68, 305-313. http://dx.doi.org/10.1016/j.carbpol.2006.12.004

Kim, H. S., Choi, H. S., Kim, B. Y. \& Baik, M. Y. (2011). Carbohydrate Polymers, 83, 755-761. http://dx.doi.org/10.1016/j.carbpol.2010.08.048

Kittipongpatana, $\quad$ o. $\quad$ S., Chaichanasak, N., Kanchongkittipoan, S., Panturat, A., Taekanmark, T., \& Kittipongpatana, N. (2006). Starch, 58 587-589. http://dx.doi.org/10.1002/star.200600528

Lawal, 0. S., Lechner, M. D., Hartmann, B., \& Kulicke, W.-M. (2007). Starch - Stärke, 59, 224-233. http://dx.doi.org/10.1002/star.200600594

Mason, W. R. (2003). Starch Use in Foods. In Starch: Chemistry and Technology, Third Edition, Chapter 20, 746.

Mishra, S., Mukul, A., Sen, G., \& Jha, U. (2011). International Journal of Biological Macromolecules, 48, 106-111. http://dx.doi.org/10.1016/i.ijbiomac.2010.10.004 PMid:20951725

Nabais, T., Brouillet, F., Kyriacos, S., \& Mroueh, M. (2007). European Journal of Pharmaceutics and Biopharmaceutics, 65, 371-378. http://dx.doi.org/10.1016/i.ejpb.2006.12.001 PMid: 17275270

Nagasawa, N., Yagi, T., Kume, T., \& Yoshii, F. (2004). Carbohydrate Polymers, 58, 109-113. http://dx.doi.org/10.1016/j.carbpol.2004.04.021

Prochaska, K., Konowal, E., Sulej-Chojnacka, J., Lewandowicz, G. (2009). Colloids and Surfaces B: Biointerfaces, 74(1), 238-243. http://dx.doi.org/10.1016/i.colsurfb.2009.07.034 PMid:19734024

Qiu, H., \& He, L. (1999). Polymers for Advanced Technologies, 10, 468-472.

http://dx.doi.org/10.1002/(SICI)1099-1581(199907)10:7 <468::AID-PAT898>3.0.CO;2-W

Sen, G., \& Pal, S. (2009). Journal of Applied Polymer Science, 114, 2798-2805. http://dx.doi.org/10.1002/app.30762

Teramoto, N., Motoyama, T., Yosomia, R., \& Shibata, M. (2003). European Polymer Journal, 39, 255-263. http://dx.doi.org/10.1016/S0014-3057(02)00199-4

Tolvanen, P., Mäki-Arvela, P., Sorokin, A. B., Salmi, T., Murzin, D. Y. (2009). Chemical Engineering Journal, 154, 52-59. http://dx.doi.org/10.1016/j.cej.2009.02.001

Wang, J., Zhou, X., \& Xiao, H. (2013). Carbohydrate Polymers, 94, 749-754. http://dx.doi.org/10.1016/i.carbpol.2013.01.036 PMid:23544629.

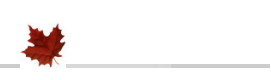

